\title{
Magnetic ground state of the one-dimensional ferromagnetic chain compounds $M(\mathrm{NCS})_{2}(\text { thiourea })_{2}(M=\mathrm{Ni}, \mathrm{Co})$
}

\author{
S. P. M. Curley $\odot,{ }^{1}$ R. Scatena $\odot,{ }^{2,3}$ R. C. Williams, ${ }^{1}$ P. A. Goddard $\odot,{ }^{1, *}$ P. Macchi $\odot,{ }^{4,5,3}$ T. J. Hicken $\odot,{ }^{6}$ T. Lancaster $\odot,{ }^{6}$ \\ F. Xiao, ${ }^{7}$ S. J. Blundell $\odot,{ }^{2}$ V. Zapf $\odot,{ }^{8}$ J. C. Eckert, ${ }^{9}$ E. H. Krenkel, ${ }^{9}$ J. A. Villa ${ }^{10}$ M. L. Rhodehouse, ${ }^{10}$ and J. L. Manson ${ }^{10, \dagger}$ \\ ${ }^{1}$ Department of Physics, University of Warwick, Gibbert Hill Road, Coventry CV4 7AL, United Kingdom \\ ${ }^{2}$ Department of Physics, Clarendon Laboratory, University of Oxford, Parks Road, Oxford OX1 3PU, United Kingdom \\ ${ }^{3}$ Department of Chemistry and Biochemistry, University of Bern, 3012 Bern, Switzerland \\ ${ }^{4}$ Department of Chemistry, Materials, and Chemical Engineering, Polytechnic of Milan, Milan 20131, Italy \\ ${ }^{5}$ Istituto Italiano di Tecnologia, Center for Nano Science and Technology CNST@ polimi, Milan 20133, Italy \\ ${ }^{6}$ Department of Physics, Durham University, South Road, Durham DH1 3LE, United Kingdom \\ ${ }^{7}$ Laboratory for Neutron Scattering and Imaging, Paul Scherrer Institut, CH-5232 Villigen PSI, Switzerland \\ ${ }^{8}$ National High Magnetic Field Laboratory, Materials Physics and Applications Division, Los Alamos National Laboratory, \\ Los Alamos, New Mexico 87545, USA \\ ${ }^{9}$ Physics Department, Harvey Mudd College, Claremont, California 91711, USA \\ ${ }^{10}$ Department of Chemistry and Biochemistry, Eastern Washington University, Cheney, Washington 99004, USA
}

(Received 26 November 2020; accepted 3 February 2021; published 3 March 2021)

\begin{abstract}
The magnetic properties of the two isostructural molecule-based magnets-Ni(NCS $)_{2}$ (thiourea) $)_{2}, S=1$ [thiourea $\left.=\mathrm{SC}\left(\mathrm{NH}_{2}\right)_{2}\right]$ and $\mathrm{Co}(\mathrm{NCS})_{2}(\text { thiourea })_{2}, S=3 / 2$ - are characterized using several techniques in order to rationalize their relationship with structural parameters and to ascertain magnetic changes caused by substitution of the spin. Zero-field heat capacity and muon-spin relaxation measurements reveal low-temperature long-range ordering in both compounds, in addition to Ising-like $(D<0)$ single-ion anisotropy $\left(D_{\mathrm{Co}} \sim-100 \mathrm{~K}\right.$, $\left.D_{\mathrm{Ni}} \sim-10 \mathrm{~K}\right)$. Crystal and electronic structure, combined with dc-field magnetometry, affirm highly quasi-onedimensional behavior, with ferromagnetic intrachain exchange interactions $J_{\mathrm{Co}} \approx+4 \mathrm{~K}$ and $J_{\mathrm{Ni}} \sim+100 \mathrm{~K}$ and weak antiferromagnetic interchain exchange, on the order of $J^{\prime} \sim-0.1 \mathrm{~K}$. Electron charge- and spin-density mapping reveals through-space exchange as a mechanism to explain the large discrepancy in $J$-values despite, from a structural perspective, the highly similar exchange pathways in both materials. Both species can be compared to the similar compounds $M \mathrm{Cl}_{2}$ (thiourea) ${ }_{4}, M=\mathrm{Ni}$ (II) (DTN) and $\mathrm{Co}$ (II) (DTC), where DTN is known to harbor two magnetic-field-induced quantum critical points. Direct comparison of DTN and DTC with the compounds studied here shows that substituting the halide $\mathrm{Cl}^{-}$ion for the $\mathrm{NCS}^{-}$ion results in a dramatic change in both the structural and magnetic properties.
\end{abstract}

DOI: 10.1103/PhysRevMaterials.5.034401

\section{INTRODUCTION}

Constraining magnetic moments to lie and interact in one-dimensional chains or two-dimensional planes has, over the years, been an area of continued interest within the magnetism community [1-3]. The reduced dimensionality generally serves to enhance quantum fluctuations, leading to the material hosting an array of exotic quantum ground states [4,5]. Several classes of low-dimensional materials can exhibit an order-to-disorder transition driven by an external tuning parameter, such as a magnetic field, that pushes the system though a quantum critical point (QCP) [6,7].

Considering the case of one-dimensional (1D) materials, a notable system is the ferromagnetically coupled effective $S=1 / 2$ chain material $\mathrm{CoNb}_{2} \mathrm{O}_{6}$. Applying a magnetic field transverse to $\mathrm{Co}$ (II) Ising-axis pushes the system through a

\footnotetext{
*p.goddard@warwick.ac.uk

†jmanson@ewu.edu
}

QCP, as it moves from a ferromagnetic (FM) ordered ground state to a disordered quantum paramagnetic phase $[8,9]$. The existence of a QCP in 1D chains is not exclusive to the case of FM coupling. The $S=1$ antiferromagnetic (AFM) chain material $\mathrm{NiCl}_{2}$ (thiourea $)_{4}(\mathrm{DTN})$, where thiourea $=\mathrm{SC}\left(\mathrm{NH}_{2}\right)_{2}$, passes through two field-induced QCPs, at least one of which belongs to the universality class of a Bose-Einstein condensate (BEC) [10,11].

In terms of physically realizing such systems, the use of organic ligands has proven highly effective in constructing crystal architectures that readily promote low-dimensional magnetic behavior [12-16]. A selection of recently published materials showcases the ability to test the limits of the theoretical understanding of $S=1 / 2$ and 1 chain materials [17-19]. To achieve quasi-one-dimensional (Q1D) behavior, the choice of intrachain bridging-ligand is a decisive one, as it ultimately determines the sign and strength of the intrachain exchange interaction $(J)$. Nonbridging ligands also play an important role in promoting Q1D behavior, keeping adjacent chains well separated and mitigating interactions due to interchain 


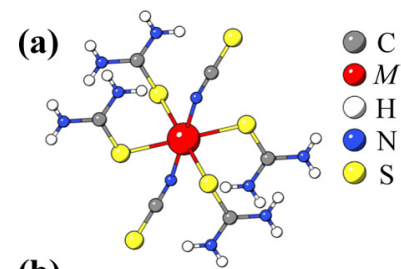

(b)

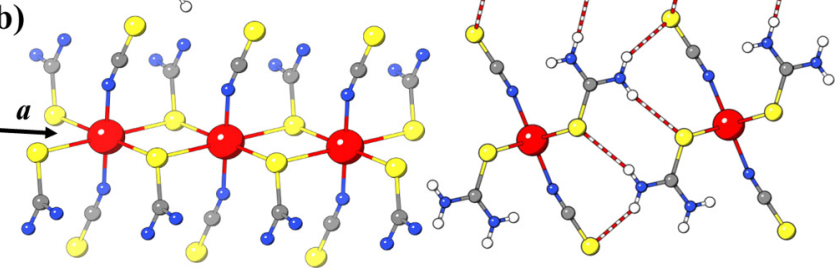

FIG. 1. Structure of $M(\mathrm{NCS})_{2}(\text { thiourea })_{2}, \quad$ where $M=\mathrm{Ni}^{2+}, \mathrm{Co}^{2+}$. (a) Local $M^{2+}$ octahedral environment. (b) Two $M-\mathrm{S}-M$ bonds form bibridge chains that propagate along the crystallographic $a$-axis. (c) H-bonding (striped bonds) between adjacent chains within the $b c$-plane. The structure is shown for $M=\mathrm{Co}^{2+}$ at $T=100 \mathrm{~K}$.

exchange $\left(J^{\prime}\right)[15,16]$. Characterizing both $J$ and $J^{\prime}$ is therefore paramount to establishing how the crystal structure influences the magnetism in Q1D systems. The ultimate goal of this work is to move toward the construction of bespoke magnetic materials, where the magnetic properties can be chemically tuned from the point of synthesis. The work also provides an avenue to study how structural properties influence the observed ground state, and the possible emergence of quantum-critical behavior, in low-dimensional magnetic systems.

We therefore turned our attention to the two isostructural coordination polymers: $\mathrm{Co}(\mathrm{NCS})_{2}$ (thiourea $)_{2}, S=3 / 2$, and the $S=1$ analog $\mathrm{Ni}(\mathrm{NCS})_{2}$ (thiourea) $)_{2}$, both of which were first synthesized some time ago [20-22]. We present here a comprehensive study of the magnetic properties of the two compounds. The isostructural architecture of the two compounds, outlined below, permits us to investigate the effect that the choice of transition-metal ion has upon both the sign and strength of $J$ and $J^{\prime}$, both of which govern the dimensionality of the system. An additional reason to study the properties of these materials is to establish their connection to known, chemically similar quantum magnets, such as the 1D chain DTN [23]. In the chosen materials, the transition-metal ion $(M)$ sits in a distorted $M \mathrm{~S}_{4} \mathrm{~N}_{2}$ octahedral environment, pictured in Fig. 1(a), suggesting the compounds likely possess a nonzero single-ion anisotropy (SIA) parameter $(D)$ and rhombic anisotropy term $(E)$. Along the crystallographic $a$ axis, adjacent $M$-ions form chains through sulfide ions on two thiourea molecules forming two S-bridge pathways as shown in Fig. 1(b). The $M-\mathrm{S}-M$ bond angles are close to $90^{\circ}$; therefore, according to the Goodenough-Kanamori-Anderson rules [24,25], these chain compounds represent promising platforms to investigate Q1D FM behavior. The magnetic properties of both compounds within an applied magnetic field $\left(\mu_{0} H\right)$ can be summarized by the following Hamiltonian:

$$
\begin{aligned}
\mathcal{H}= & -J \sum_{\langle i, j\rangle} \hat{\mathbf{S}}_{i} \cdot \hat{\mathbf{S}}_{j}-J^{\prime} \sum_{\left\langle i, j^{\prime}\right\rangle} \hat{\mathbf{S}}_{i} \cdot \hat{\mathbf{S}}_{j^{\prime}}+D \sum_{i}\left(\hat{\mathbf{S}}_{i}^{z}\right)^{2} \\
& +E \sum_{i}\left[\left(\hat{\mathbf{S}}_{i}^{x}\right)^{2}-\left(\hat{\mathbf{S}}_{i}^{y}\right)^{2}\right]+\mu_{\mathrm{B}} \mu_{0} \sum_{i} \mathbf{H} \cdot \mathbf{g} \cdot \hat{\mathbf{S}}_{i},
\end{aligned}
$$

where $\hat{\mathbf{S}}_{i}$ is the spin of each ion $i,\langle i, j\rangle$ denotes a sum over nearest neighbors, and a primed index in the summation describes the interaction with a nearest neighbor in an adjacent chain; $J>0$ corresponds to FM interactions. Here, $\mathbf{g}=$ $\operatorname{diag}\left(g_{x}, g_{y}, g_{z}\right)$ is a tensor of $g$-factors where diagonal components are not necessarily equal. While the triclinic structure of both materials is permissive of an $E$-term, the equatorial $M \mathrm{~S}_{4}$ environment (discussed in detail below) is only slightly distorted. As the departure from octahedral symmetry is limited, any $E$-term is expected to be small, as observed in similar $\mathrm{Ni}(\mathrm{II})$ and $\mathrm{Co}(\mathrm{II})$ complexes [26-28].

Several methods were employed to investigate the electronic and magnetic properties of the two compounds in an attempt to ascertain the sign and magnitude of the dominant terms in Eq. (1): $J, J^{\prime}$, and $D$. To identify likely magnetic exchange pathways, both crystal and electronic structures were inspected using multipolar model refinement of high-resolution single-crystal x-ray diffraction data. Chemical bonding analysis was performed on the electron charge-density distributions obtained from x-ray diffraction and density functional theory (DFT) calculations, and compared with calculated spin-density maps to highlight the role of the magnetic ions. Heat capacity combined with muonspin relaxation measurements revealed magnetic ordering and elucidated the magnitude of the SIA. Quasistatic dc-field magnetometry measurements, in combination with DFT calculations of the exchange coupling constants, helped illustrate a coherent picture of the magnetic ground state.

\section{RESULTS}

\section{A. Crystal structure}

Crystallization of $\mathrm{Co}(\mathrm{NCS})_{2}$ (thiourea $)_{2}$ and $\mathrm{Ni}(\mathrm{NCS})_{2}$ (thiourea) $)_{2}$ coordination polymers resulted in needle-shaped single crystals where single-crystal x-ray diffraction (SCXRD) measurements revealed twinning in both. $\mathrm{Co}(\mathrm{NCS})_{2}$ (thiourea) $)_{2}$ could, however, also be obtained with thin-sheet morphology; these crystals showed clean single-crystal diffraction frames. Needlelike $\mathrm{Ni}(\mathrm{NCS})_{2}$ (thiourea $)_{2}$ crystals were irremediably affected by twinning, and attempts to recrystallize different morphologies proved unsuccessful. Both compounds crystallize into a triclinic structure with the space-group $P \overline{1}$; selected structural refinement parameters are listed in Table SIV of the supplemental information (SI) (as are the experimental details); see [29].

High-quality $\mathrm{Co}(\mathrm{NCS})_{2}$ (thiourea $)_{2}$ single crystals allowed x-ray diffraction data to be collected up to $d_{\min }=0.50 \AA$, which, in combination with low temperatures $(T=100 \mathrm{~K})$, allowed the refinement of the crystal structure, but also of the aspherical electron-charge-density distribution, presented below. The Co atom resides on an inversion center and lies in the middle of an octahedron built by pairs of $\mathrm{Co}-\mathrm{N}$ [2.0226(4) $\mathrm{A}], \mathrm{Co}-\mathrm{S}[2.5523(1) \AA]$, and $\mathrm{Co}-\mathrm{S}$ [2.5972(1) $\AA]$ coordination bonds. As a result of these bond lengths, the octahedral environment is axially compressed along the $\mathrm{N}-\mathrm{Co}-\mathrm{N}$ axis. The $\mathrm{S}-\mathrm{Co}-\mathrm{S}$ angles within the equatorial plane $\left[84.19(1)^{\circ}\right.$ and $\left.95.81(1)^{\circ}\right]$ and the angle between the octahedron axis and the equatorial plane $\left[96.56(1)^{\circ}\right]$ show 
that the $\mathrm{Co}$ (II) octahedra are slightly distorted. $\mathrm{A} \mathrm{Co}-\mathrm{S}-\mathrm{Co}$ angle of $95.81(1)^{\circ}$ defines the geometry of the two $\mathrm{Co}-\mathrm{S}-$ Co bridges that make up the polymeric chain. The polymeric chains are packed together in the $b c$-plane by $\mathrm{H}$-bonds in the range $3.43-3.47 \AA$ and classify as weakly interacting.

Single-crystal $\mathrm{x}$-ray diffraction data for $\mathrm{Ni}(\mathrm{NCS})_{2}$ (thiourea) $)_{2}$ were measured at $T=173 \mathrm{~K}$ up to $d_{\min }=0.70 \AA$ with a second twinning component of 27.7(1)\% observed in the crystal. Analogous to the structure of $\mathrm{Co}(\mathrm{NCS})_{2}$ (thiourea $)_{2}$, the $\mathrm{Ni}$ atom sits on the inversion center in the middle of an octahedron built by two of each $\mathrm{Ni}-\mathrm{N}\left[\begin{array}{ll}1.997(2) & \AA\end{array}\right], \mathrm{Ni}-\mathrm{S}[2.5069(6) \AA]$, and $\mathrm{Ni}-\mathrm{S}$ $\left[\begin{array}{ll}2.5517(6) & \AA\end{array}\right]$ coordination bonds. The $\mathrm{S}-\mathrm{Ni}-\mathrm{S}$ angles within the equatorial plane $\left[83.93(2)^{\circ}\right.$ and $\left.96.07(2)^{\circ}\right]$ and the angle between the octahedron axis and the equatorial plane $\left[97.20(7)^{\circ}\right]$ also show a slight distortion to the $\mathrm{Ni}(\mathrm{II})$ octahedra. A Ni-S-Ni angle of $96.07(2)^{\circ}$ defines the geometry of two $\mathrm{Ni}-\mathrm{S}-\mathrm{Ni}$ bridges along the polymeric chain. $\mathrm{H}$-bonds pack the chains in the $b c$-plane with distances between the donor and acceptor within the range 3.43-3.45 $\AA$, which again classify as weakly bonded.

The experimentally determined structural parameters of $\mathrm{Co}(\mathrm{NCS})_{2}$ (thiourea $)_{2}$ and $\mathrm{Ni}(\mathrm{NCS})_{2}$ (thiourea $)_{2}$ are very similar to one another. This similarity is found also from periodic DFT optimization of the measured geometries (see Table SVI [29]), which returned an even tighter correspondence between bond lengths and angles in the two species.

\section{B. Muon-spin relaxation}

Zero-field positive-muon-spin-relaxation $\left(\mathrm{ZF} \mu^{+} \mathrm{SR}\right)$ measurements on $M(\mathrm{NCS})_{2}$ (thiourea $)_{2}$ were performed, with example spectra spanning the measured temperature range shown in Fig. 2. The spectra measured for $\mathrm{Co}(\mathrm{NCS})_{2}$ (thiourea $)_{2}$ show no oscillations in the asymmetry, but they consist of two exponentially relaxing components, one with a large relaxation rate, found to be constant over the entire temperature range, and one with a smaller relaxation rate that dominates at later times; see the SI [29] and Ref. [30] for fit details. The large relaxation rate is often observed in coordination polymers of this type and has been ascribed to a class of muon sites that are not well coupled to the magnetism (hence its temperature independence) that are realized close to electron density (e.g., double bonds, aromatic rings, etc.) $[31,32]$.

The temperature evolution of the smaller relaxation rate $\left(\lambda_{1}\right)$ is shown in Fig. 3. It exhibits a peak around $8 \mathrm{~K}$, indicating a phase transition from a magnetically ordered to a disordered state, in good agreement with heat-capacity and magnetometry measurements discussed below. The lack of oscillations at low temperature and the observed exponential relaxation suggest that the system is dominated by dynamic fluctuations on the muon timescale, such that coherent precession of the muon-spin is not measured. This has been noted in several coordination polymer magnets containing $\mathrm{Fe}^{2+}$ [33], $\mathrm{Mn}^{2+}$ [34], and (in some cases) $\mathrm{Ni}^{2+}$ [35], where the sizable magnetic moment can lead to a large, fluctuating distribution of local magnetic fields at the muon sites. (In contrast, coordination polymer magnets containing $\mathrm{Cu}^{2+}$ often show oscillations in the ordered regime [31].) The observed

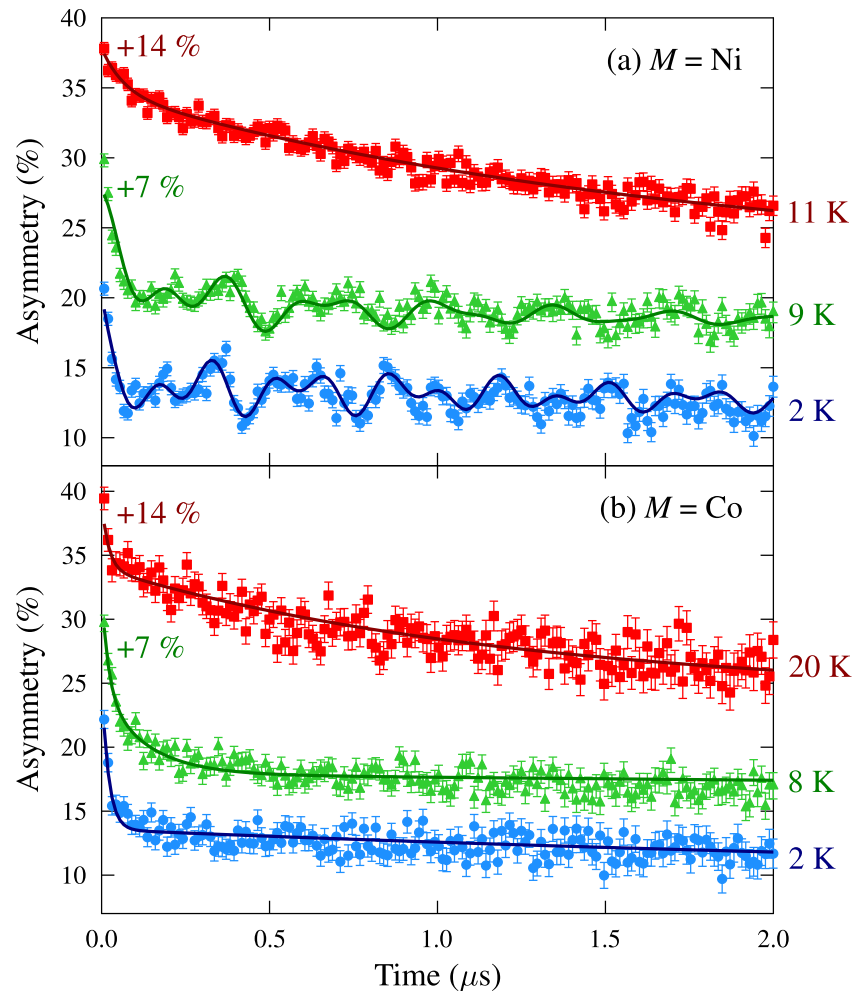

FIG. 2. $\mathrm{ZF} \mu^{+} \mathrm{SR}$ spectra measured on (a) $\mathrm{Ni}(\mathrm{NCS})_{2}$ (thiourea $)_{2}$ and (b) $\mathrm{Co}(\mathrm{NCS})_{2}$ (thiourea $)_{2}$. Data are presented with a vertical offset where needed for clarity. Fits shown as detailed in the Supplemental Material [29].

exponential relaxation suggests that the sample is in the fastfluctuation regime below $T_{\mathrm{c}}$, where the relaxation rate varies as $\lambda \propto \Delta^{2} \tau$. Here $\Delta$ is the variance of the field distribution sampled by the muons, and $\tau$ is the fluctuation time. The variance $\Delta$ varies with the size of the local magnetic field,

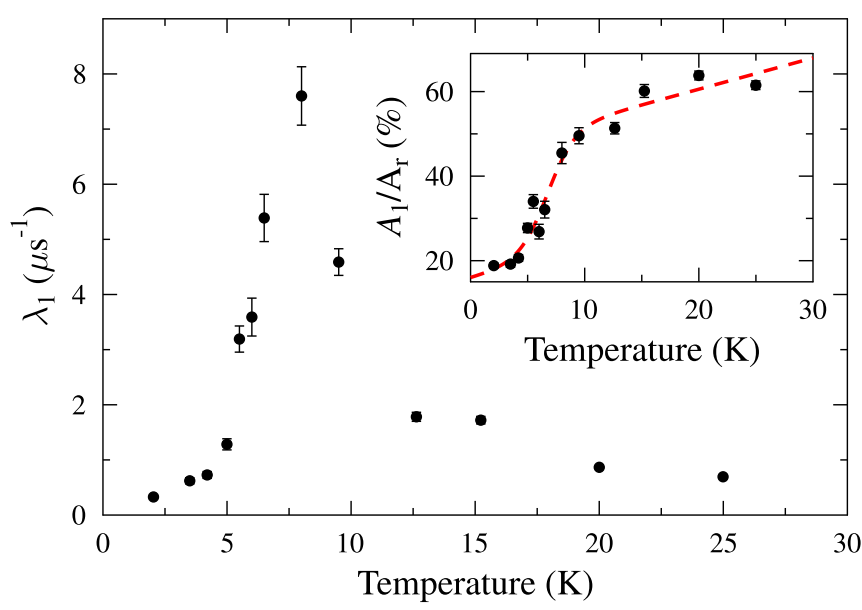

FIG. 3. Temperature evolution of the small relaxation rate $\left(\lambda_{1}\right)$ extracted through fitting $\mathrm{ZF} \mu^{+} \mathrm{SR}$ asymmetry spectra measured on $\mathrm{Co}(\mathrm{NCS})_{2}$ (thiourea $)_{2}$. The inset shows the temperature evolution of the ratio of the amplitude of the component with a small relaxation rate $\left(A_{1}\right)$ to the total relaxing asymmetry $\left(A_{\mathrm{r}}\right)$. The line is a guide to the eye. 


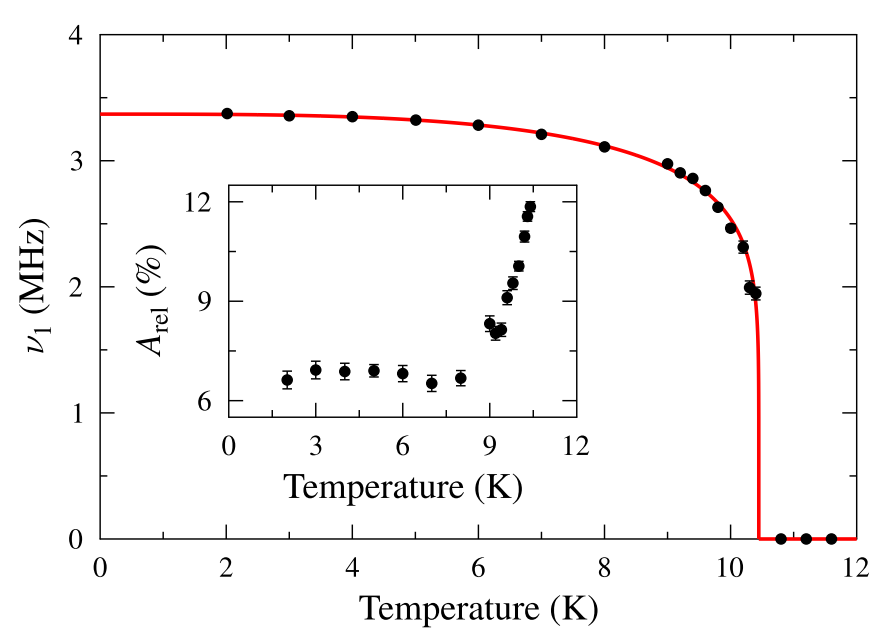

FIG. 4. Temperature evolution of the smallest oscillation frequency $\left(v_{1}\right)$ observable in the $\mathrm{ZF} \mu^{+} \mathrm{SR}$ asymmetry spectra measured on $\mathrm{Ni}(\mathrm{NCS})_{2}$ (thiourea $)_{2}$, with a fit to Eq. (2). The inset shows the temperature evolution of the total relaxing asymmetry $\left(A_{\text {rel }}\right)$ from the same measurements.

so we might expect materials with larger moments to lead to larger relaxation rates. In this scenario, the relaxation rate will scale faster with moment than the oscillation frequency, and therefore a higher moment could prevent the observation of coherent precession. This could explain the observed lack of oscillations in this system compared to similar materials with smaller moments. It is also possible that the lack of oscillations reflects a greater propensity for Co-based systems to adopt magnetic structures that yield inhomogeneous local field distributions or that give dynamic fluctuations in the muon $(\mathrm{MHz})$ time frame.

Further evidence for a transition around $8 \mathrm{~K}$ comes from the rapid change in amplitude of the small relaxation rate component $\left(A_{1}\right)$, as shown in the inset of Fig. 3. (This effect has also been observed in similar materials close to the transition temperature [33-35].) Significant in this case is that both this change and the peak in $\lambda$ persist over a relatively wide range of temperature, extending at least $2-3 \mathrm{~K}$ below $T_{\mathrm{c}}$. This suggests that the phase transition is extended in temperature on the $\mathrm{MHz}$ timescale with an onset of dynamics occurring in the ordered regime above $5 \mathrm{~K}$.

In contrast to the $\mathrm{Co}$ material, $\mathrm{ZF} \mu^{+} \mathrm{SR}$ spectra for $\mathrm{Ni}(\mathrm{NCS})_{2}$ (thiourea $)_{2}$ show oscillations in the asymmetry for temperatures $T \lesssim 10.4 \mathrm{~K}$, indicating coherent muon-spin precession consistent with long-range magnetic order. Two oscillation frequencies [in a constant ratio $v_{2} / v_{1}=1.754(1)$ ] were observable over the whole temperature range, with a third $v_{3} \simeq 3 v_{1}$ only observable for $T \lesssim 7 \mathrm{~K}$. This indicates three distinct muon stopping sites in this material.

By fitting these spectra as seen in Fig. 2(b) (see the SI [29] for fit details), the frequencies were extracted. The smallest frequency was fitted to

$$
v_{i}(T)=v_{i}(0)\left[1-\left(\frac{T}{T_{\mathrm{N}}}\right)^{\delta}\right]^{\beta},
$$

as seen in Fig. 4. It was found that $v_{1}(0)=3.37(2) \mathrm{MHz}$ [corresponding to a local magnetic field of 24.1(1) $\mathrm{mT}$ at the muon-spin site at zero temperature], $\delta=3.2(3), \beta=0.14(3)$, and $T_{\mathrm{N}}=10.4(1) \mathrm{K}$. This value of $\beta$ is consistent with a system dominated by $2 \mathrm{D}$ magnetic fluctuations, being very close to the value for the $2 \mathrm{D}$ Ising model, $\beta=1 / 8$ [36]. This transition temperature is also supported by the behavior of relaxation rates of the oscillating components, which both show a peak between $T=10.3$ and $10.4 \mathrm{~K}$. These rates diverge when approached from below, indicating a critical slowing down of magnetic fluctuations, which often occurs in the proximity of a magnetic transition.

The disappearance of the third frequency, in combination with the increase in the relaxing asymmetry $\left(A_{\text {rel }}\right)$ for $T \gtrsim$ $7 \mathrm{~K}$, seen in the inset of Fig. 4, is evidence for the onset of the magnetic transition in this material, suggesting again a broad phase transition taking place in the region $7 \lesssim T \lesssim 10.4 \mathrm{~K}$ on the muon timescale. For $T \gtrsim 10.4 \mathrm{~K}$, no oscillations are observable in the asymmetry spectra, consistent with the system being magnetically disordered.

\section{Heat capacity}

Zero-field heat capacity $(C)$ was measured as a function of temperature for a single-crystal of $\mathrm{Co}(\mathrm{NCS})_{2}$ (thiourea $)_{2}$ and a polycrystalline pressed pellet of $\mathrm{Ni}(\mathrm{NCS})_{2}$ (thiourea $)_{2}$. To isolate the low-temperature magnetic heat capacity $\left(C_{\mathrm{mag}}\right)$, the high-temperature $(T \gtrsim 30 \mathrm{~K})$ contribution was reproduced using a phenomenological model containing both Debye and Einstein phonon modes and subtracted as a background $[29,37]$. For both compounds, a $\lambda$-peak in $C_{\text {mag }}$ at low temperatures, seen in Fig. 5(a), is indicative of a transition to a magnetically ordered state, giving ordering temperatures of $T_{c}=6.82(5)$ and $10.5(1) \mathrm{K}$ for the $\mathrm{Co}$ and $\mathrm{Ni}$ species, respectively, in excellent agreement with $\mathrm{ZF} \mu^{+} \mathrm{SR}$.

$\mathrm{Co}(\mathrm{NCS})_{2}$ (thiourea $)_{2} C_{\text {mag }}$ data possess a notably sharper $\lambda$-peak compared to $\mathrm{Ni}(\mathrm{NCS})_{2}$ (thiourea $)_{2}$. Single-crystal magnetometry for the Co species, discussed below, provides the reason for this. The magnetometry data indicate an AFM ground state, where the spins possess a strong Ising-like SIA. For a Q1D chain of Ising spins, a broad hump in $C_{\mathrm{mag}}$ is expected as the reduced dimensionality causes the buildup of spin-spin correlations at temperatures above the transition temperature $[38,39]$. The sharp nature of the peak points to $2 \mathrm{D}$ or $3 \mathrm{D}$ Ising-like long-range ordering within the material. The measured response of $C_{\text {mag }}(T)$ was not well captured by simulations of $C_{\mathrm{mag}}(T)$ for the 2D Ising model [40,41] (see Fig. S2 [29]). This would imply that the secondary exchanges along $b$ and $c$ are similar in magnitude, which is also supported by the DFT calculations (below) and indicates $n^{\prime}=4$ next nearest neighbors.

The magnetic entropy per mole $\left(S_{\mathrm{mag}}\right)$ for both compounds can be determined using

$$
S_{\mathrm{mag}}(T)=\int_{0}^{T} d T^{\prime} \frac{C_{\mathrm{mag}}\left(T^{\prime}\right)}{T^{\prime}},
$$

where it is assumed that $C_{\mathrm{mag}}=0$ at $T=0 \mathrm{~K}$. The results are shown in the inset of Fig. 5(a). For Ni(NCS) $)_{2}$ (thiourea $)_{2}$, $S_{\text {mag }}$ fully recovers to $R \ln (3)$ ( $R$ is the ideal gas constant) as expected for an $S=1$ ion. In contrast, $\mathrm{Co}(\mathrm{NCS})_{2}$ (thiourea) $)_{2}$ shows an initial sharp upturn before a broad rise to a plateau at $R \ln (2)$ around $30 \mathrm{~K}$. Electronic structure calculations reveal 


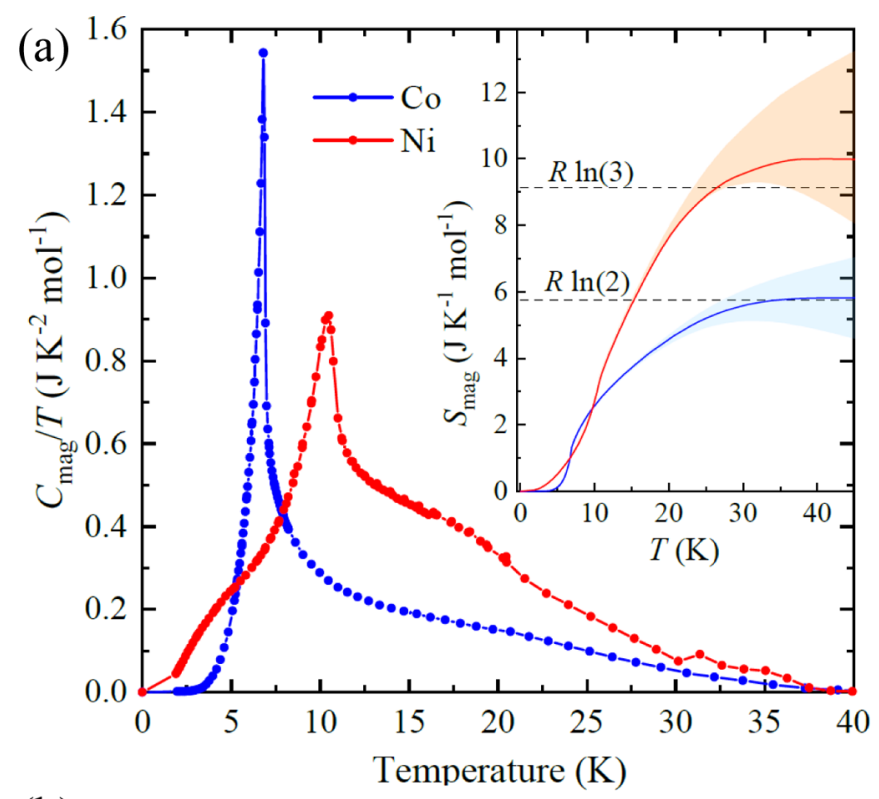

(b)

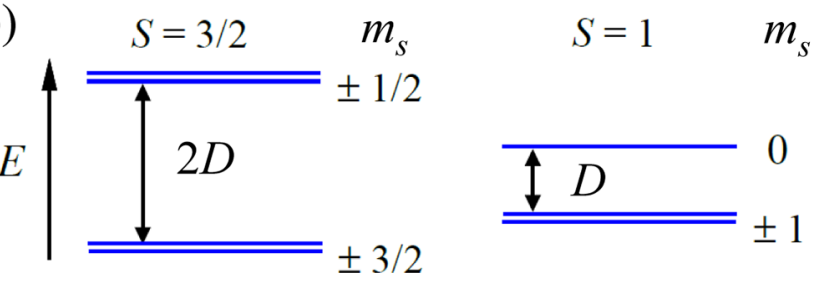

FIG. 5. (a) Zero-field magnetic heat capacity $\left(C_{\text {mag }}\right)$ divided by temperature $(T)$ and plotted as a function of $T$. The inset shows the resultant magnetic entropy $\left(S_{\mathrm{mag}}\right)$, where the shaded regions are representative of errors introduced by the uncertainty in the amplitudes of the high-temperature fit components. (b) Energy $(E)$ level diagrams for $S=3 / 2$ and 1 moments within octahedral environments with easy-axis single-ion anisotropy $(D<0)$, where $m_{s}$ is the eigenvalue of the spin operator $S_{z}$.

that the $\mathrm{Co}$ ion sits in the high-spin $\mathrm{Co}^{2+}$ state. The spin configuration consists of two Kramer doublets split by an energy gap of magnitude $2 D$ [see Fig. 5(b)]. For an $S=3 / 2$ ion, we expect $S_{\text {mag }}$ to recover to $R \ln (4)$ in accordance with $R \ln (2 S+1)$. A recovery to $R \ln (2)$ is indicative of a system exhibiting large SIA, which keeps the two Kramer doublets well separated, as illustrated in Fig. 5(b), suggesting that the system can be well approximated using an effective spin-half approach within the low-temperature regime. We therefore expect $D \sim-100 \mathrm{~K}$ as seen in similar easy-axis Co(II) complexes [28].

The behavior of the Ni species was investigated further by measuring $C_{\text {mag }}$ in applied magnetic fields up to $9 \mathrm{~T}$, as shown in Fig. 6 . The $\lambda$-peak is initially suppressed in field, suggesting the ground state is AFM in nature. At higher fields, the ordering peak ceases to be resolvable, and the broad hump shifts to higher temperatures reflecting the Schottky-like response to the field-induced Zeeman splitting of the ground-state energy levels. The inset of Fig. 6 shows that $S_{\text {mag }}$ fully recovers to $R \ln (3)$ on warming above about $40 \mathrm{~K}$ in zero field and $50 \mathrm{~K}$ at $9 \mathrm{~T}$, indicating the level splitting is completely overcome by these temperatures. This is consistent with $|D| \sim 10 \mathrm{~K}$, which is typical for $\mathrm{Ni}^{2+}$ ions in similar local environments $[42,43]$.

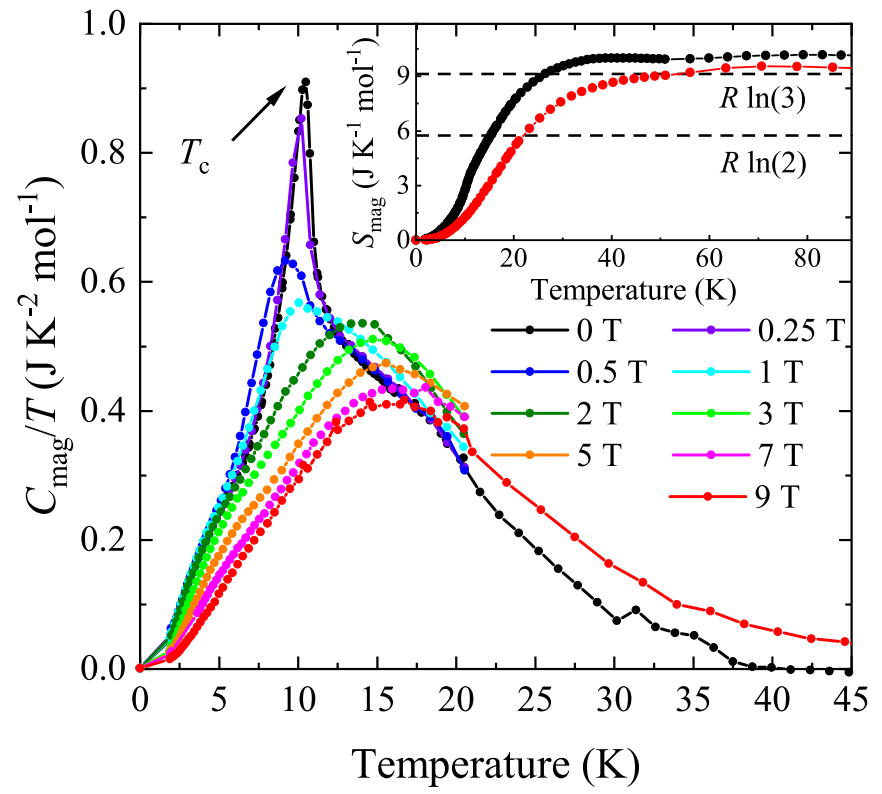

FIG. 6. Magnetic heat capacity $\left(C_{\mathrm{mag}}\right)$ divided by temperature $(T)$ and plotted as a function of $T$ for a polycrystalline pressed pellet of $\mathrm{Ni}(\mathrm{NCS})_{2}$ (thiourea $)_{2}$ measured in applied fields up to $9 \mathrm{~T}$. The zero-field transition temperature to a magnetically ordered state is indicated at $T_{c}$. The inset shows that magnetic entropy $\left(S_{\mathrm{mag}}\right)$ recovers to $R \ln (3)$ at 0 and $9 \mathrm{~T}$, as expected for an $S=1$ ion.

\section{Magnetometry}

Single-crystal magnetometry measurements were performed on $\mathrm{Co}(\mathrm{NCS})_{2}$ (thiourea $)_{2}$ with the external magnetic field applied along three orthogonal orientations: parallel to the chain axis $a$, perpendicular to $a$ within the $a b$-plane (denoted $b^{\prime}$ ), and normal to the $a b$-plane (denoted $c^{\prime}$ ). The angle between the magnetic field and the unique $\mathrm{N}-\mathrm{Co}-\mathrm{N}$ axis is $84^{\circ}$ for $\mu_{0} H \| a, 73^{\circ}$ for $\mu_{0} H \| b^{\prime}$, and $18^{\circ}$ for $\mu_{0} H \| c^{\prime}$.

Figure 7(a) shows that upon decreasing temperature, the magnetic susceptibility $[\chi(T)]$ for a field along all three orientations rises to a sharp peak before plateauing at low temperatures, a behavior indicative of an AFM ground state. The Fisher relation [44] estimates a transition temperature $T_{c}=6.7(1) \mathrm{K}$, in excellent agreement with heat capacity and $\mu^{+}$SR data. The $\chi(T)$ data with a field parallel to $c^{\prime}$ [red circles in Fig. 7(a)] is an order of magnitude greater than measurements made with the field along $a$ or $b^{\prime}$, verifying a strong Ising-like $(D<0)$ SIA. Due to the close proximity of $c^{\prime}$ to the unique axial $\mathrm{N}-\mathrm{Co}-\mathrm{N}$ bond, it is highly likely that the magnetic moments on the Co ions are collinear with the $\mathrm{N}-\mathrm{Co}-\mathrm{N}$ axis.

The $\chi^{-1}(T)$ data for all three field directions [see the inset of Fig. 7(a)] shows curvature persisting up to the highest measured temperature $(T=300 \mathrm{~K})$. This non-Curie-like behavior suggests that the leading energy term in the Hamiltonian is similar in size to the thermal energy in this temperature range. On the basis of our heat capacity measurements, we expect this term to be $D$. Thus we estimate $|D| \sim 100 \mathrm{~K}$ in agreement with heat capacity data and similar Co(II) complexes [28].

The large negative SIA (zero-field splitting $=2|D| \sim$ $200 \mathrm{~K}$ ) suggests that as temperature is lowered below $100 \mathrm{~K}$, 

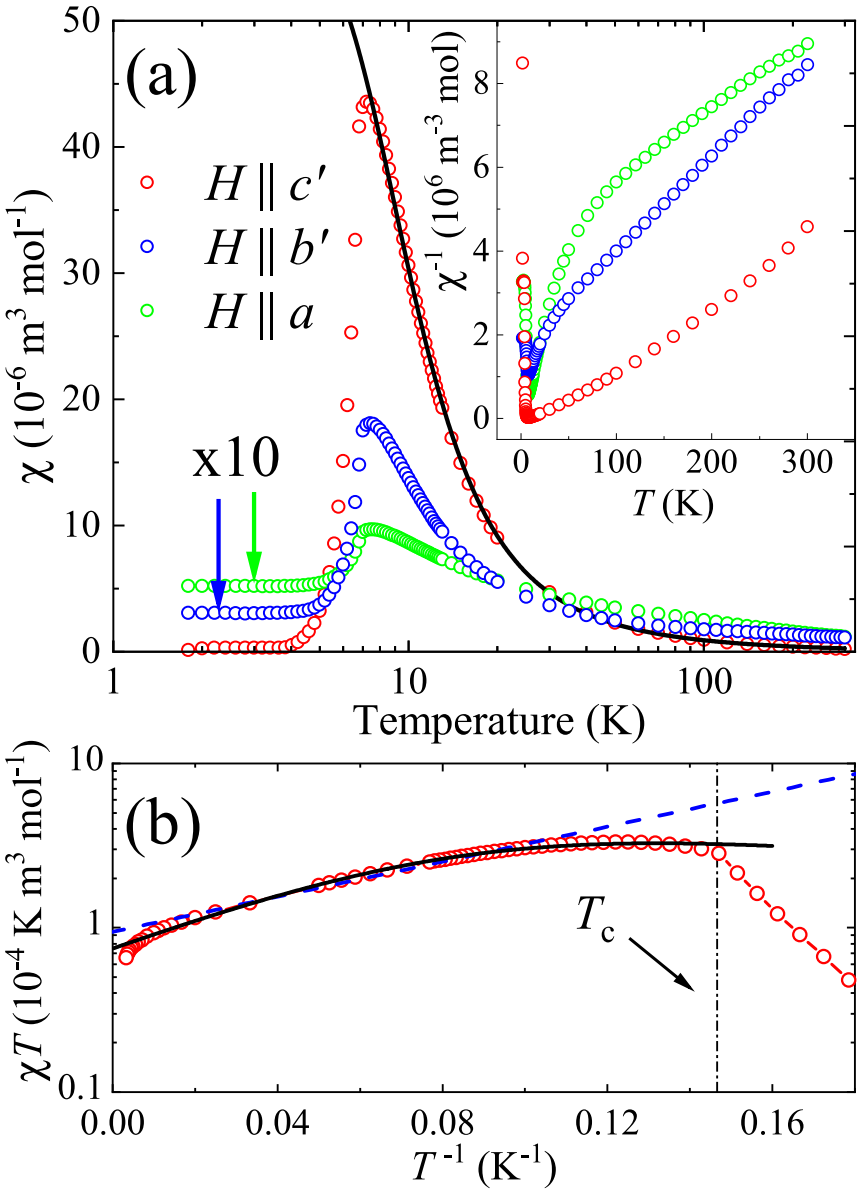

FIG. 7. Single-crystal magnetic susceptibility $\chi(T)$ data for $\mathrm{Co}(\mathrm{NCS})_{2}$ (thiourea $)_{2}$ measured at $\mu_{0} H=0.1 \mathrm{~T}$. (a) $\chi(T)$ for different field directions where data for $H \| b^{\prime}$ and $a$ are multiplied by a factor of 10 . The inset shows a plot of $\chi^{-1}(T)$ with no scaling. (b) Semilogarithmic plot of $\chi T$ against $T^{-1}$ (discussed in the text) for a field parallel to $c^{\prime}$. The dashed line is a fit to $\chi_{1 \mathrm{D}}^{\|}$(see the text). The solid line in both panels is a fit to Eq. (4) within the temperature range $10 \leqslant T \leqslant 100 \mathrm{~K}$.

a vanishingly small proportion of the spins will populate the excited doublet, and the magnetic properties can be accounted for within an effective spin-half $\left(S_{\text {eff }}=1 / 2\right)$ Ising model. This means that over the temperature range $10 \leqslant T \leqslant 100 \mathrm{~K}$, the susceptibility for $H \| c^{\prime}$ (which is close to parallel to the expected Ising axis) can be approximated by that of the ideal $1 \mathrm{D} S=1 / 2$ Ising chain $\left[\chi_{1 \mathrm{D}}^{\|}(T)\right]$ and parametrized by $J_{\text {eff }}$, the primary exchange energy in the effective $S_{\text {eff }}=1 / 2$ picture $[45,46]$. Deviations from strictly $1 \mathrm{D}$ behavior can be accounted for by introducing a mean-field correction to the susceptibility given by the expression [46]

$$
\chi_{\mathrm{mf}}=\frac{\chi_{1 \mathrm{D}}^{\|}(T)}{\left[1-\left(n^{\prime} J_{\mathrm{eff}}^{\prime}\right) \chi_{1 \mathrm{D}}^{\|}(T) / C_{1 \mathrm{D}}\right]},
$$

where $C_{1 \mathrm{D}}$ is the easy-axis Curie constant for the idealized $1 \mathrm{D}$ chain, and $J_{\text {eff }}^{\prime}$ is the interchain exchange in the effective $S_{\text {eff }}=1 / 2$ picture arising from $n^{\prime}$ interchain nearest neighbors, where $n^{\prime}=4$ for this material.

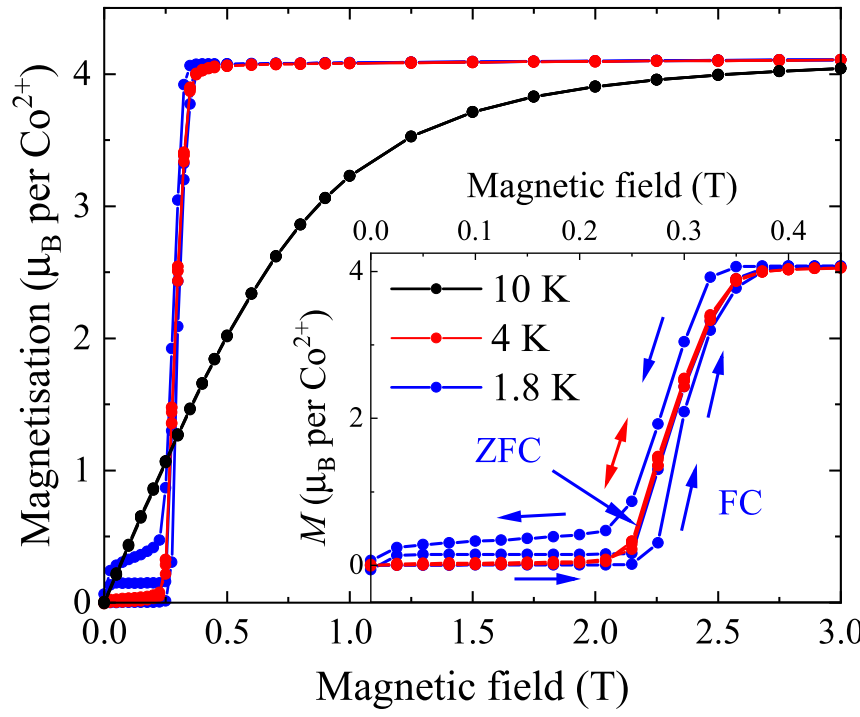

FIG. 8. Single-crystal dc-field magnetization $M(H)$ data for $\mathrm{Co}(\mathrm{NCS})_{2}$ (thiourea $)_{2}$ with the field parallel to $c^{\prime}$. The inset highlights the low-field hysteretic behavior seen at the lowest temperature. Zero-field-cooled (ZFC) and field-cooled (FC) upsweeps are indicated with arrows.

Following the analysis by Greeney et al. [46], in Fig. 7(b) we show a semilogarithmic plot of $\chi T(T)$ against $T^{-1}$ for $H \| c^{\prime}$. In this diagram, $\chi_{1 \mathrm{D}}^{\|}(T)$ is a straight line with the gradient given by the intrachain exchange $J_{\text {eff }}$ and the intercept related to $C_{1 \mathrm{D}}$. A positive slope is indicative of FM intrachain exchange $\left(J_{\text {eff }}>0\right)$. The sharp kink at $T_{\mathrm{c}}$ indicates the onset of long-range order. The data near $T_{\mathrm{c}}$ deviate from the linear response predicted by the ideal 1D Ising model (dashed line). A fit to Eq. (4) within the temperature range $10 \leqslant$ $T \leqslant 100 \mathrm{~K}\left(T<2|D|\right.$, well within the $S_{\text {eff }}=1 / 2$ regime $)$ is found to more accurately track the data [solid line, Figs. 7(a) and 7(b)], indicating the importance of the intrachain exchange interactions. The fit to Eq. (4) returned parameters of $g_{\text {eff }}=8.0(1), J_{\text {eff }}=10.4(2) \mathrm{K}$, and $J_{\text {eff }}^{\prime}=-0.31(2) \mathrm{K}$. The fit parameters are consistent with the effective $S=1 / 2$ model, in which the full $\mathrm{Co}$ (II) moment of the ground-state doublet is absorbed into the effective $g$-factor and exchange energies. The real exchange, $J$, is related to the effective value via $J=(3 / 5)^{2} J_{\text {eff }}[47,48]$, hence the values we extract are $J=$ $3.62(7) \mathrm{K}$ and $J^{\prime}=-0.12(1) \mathrm{K}$. The fit to Eq. (4) deviates from the data near the onset of long-range order. The departure at high temperatures is caused by the breakdown of the Ising model as temperatures exceed $|D| \sim 100 \mathrm{~K}$. The difference between $\chi(T)$ for the field along $b^{\prime}$ and $a$ may be due to the existence of a small $E$-term, a slight misalignment of the magnetic field, or different temperature dependencies of the effective magnetic moment along $b^{\prime}$ and $a$.

The magnetization $M(H)$ of $\mathrm{Co}(\mathrm{NCS})_{2}$ (thiourea $)_{2}$ with field parallel to $c^{\prime}$ is shown in Fig. 8. At $T \leqslant 4 \mathrm{~K}$, the induced moment is approximately zero at low fields before rapidly rising to a saturation moment of $M_{\mathrm{sat}}=4.1(1) \mu_{\mathrm{B}}$ per $\mathrm{Co}^{2+}$ ion at $\mu_{0} H_{\text {sf }}=0.29(5)$ T. We attribute this feature to spinflip behavior where the spins are rapidly polarized from their AFM ground state to an FM saturated state as the interchain 
AFM bonds are overcome by the Zeeman interaction. The step broadens and disappears at $T>4 \mathrm{~K}$.

Within the $S_{\text {eff }}=1 / 2$ model, $M_{\text {sat }}$ yields $g_{\text {eff }}=8.2(1)$, in excellent agreement with the result from fitting $\chi(T)$. The field at which the spin flip occurs can be related to the AFM interaction strength via $g_{\text {eff }} \mu_{\mathrm{B}} \mu_{0} H_{\mathrm{sf}}=S n^{\prime} J_{\text {eff }}^{\prime}$. Assuming $n^{\prime}=$ 4 , we obtain $J^{\prime}=-0.3(1) \mathrm{K}$, which is in reasonable agreement with the value extracted from $\chi(T)$. As $M(H)$ directly probes the AFM interchain bonds at low temperature, during the spin-reversal process, we expect $M(H)$ to provide us with the more trustworthy estimate of $J^{\prime}$. The inset to Fig. 8 shows hysteresis in $M(H)$ for $T<4 \mathrm{~K}$, expected for FM coupled Ising spins. This behavior is also observed in the similar FM Ising chain compound $\mathrm{Co}(\mathrm{NCS})_{2}$ (4-benzoylpyridine) $)_{2}$ (4benzoylpyridine $=\mathrm{C}_{12} \mathrm{H}_{9} \mathrm{NO}$ ) [49].

Figure 9(a) shows powder $\chi(T)$ for $\mathrm{Ni}(\mathrm{NCS})_{2}(\text { thiourea })_{2}$. Upon decreasing temperature, $\chi(T)$ rises to a peak before dropping and plateauing at low temperatures. The inset of Fig. 9(a) shows $\chi^{-1}(T)$, where a Curie-Weiss fit for $T \geqslant$ $100 \mathrm{~K}$ returns $g=2.29(1) \mathrm{K}$ and $\Theta_{\mathrm{CW}}=+42(1) \mathrm{K} \cdot \chi T(T)$ data [Fig. 9(b)] increase upon cooling from room temperature, reach a broad maximum, and then drop toward zero at $T<10 \mathrm{~K}$. At high temperature, $\chi T(T)$ data approach a flat paramagnetic value at $T \approx 300 \mathrm{~K}$, likely plateauing at $T \approx$ $300-400 \mathrm{~K}$. This corresponds to the energy scale of the largest term in the Hamiltonian $J$, such that $k_{\mathrm{B}} T \approx 2 n J$ ( $n=2$ is the number of nearest neighbors in the chain). This estimates $J \approx 75-100 \mathrm{~K}$, in good agreement with DFT calculations below. The Fisher method [44] determines $T_{c}=10.4(4) \mathrm{K}$ [Fig. 9(b)], in excellent agreement with heat capacity and $\mu^{+}$SR measurements. These observations are consistent with large FM primary exchange interactions and a considerably smaller secondary AFM exchange, leading to an AFM ground state.

Figure 9(c) shows $M(H)$ for a powder sample of $\mathrm{Ni}(\mathrm{NCS})_{2}$ (thiourea $)_{2}$. At low fields, $M(H)$ is approximately zero prior to exhibiting a sharp upturn at $\mu_{0} H_{\mathrm{sf}}=0.65(5) \mathrm{T}$, which we ascribe to spin-flip behavior. The rise of $M(H)$ slows at fields $\sim 1 \mathrm{~T}$ before increasing monotonically above $3 \mathrm{~T}$, approaching $M(H) \sim 2 \mu_{\mathrm{B}}$ per $\mathrm{Ni}^{2+}$ ion at the maximum experimentally accessible field of $7 \mathrm{~T}$. The field at which the spin flip occurs can be used to estimate the interchain magnetic exchange $J^{\prime}$ via $g \mu_{\mathrm{B}} \mu_{0} H_{\mathrm{sf}}=2 S n^{\prime} J^{\prime}$, where $n^{\prime}$ is the number of nearest interchain neighbors [50]. Taking $g=2.29(1)$, from $\chi^{-1}(T)$, and $n^{\prime}=4$, we estimate $J^{\prime}=$ $0.13(1) \mathrm{K}$.

The behavior of $M(H)$ above $\mu_{0} H_{\mathrm{sf}}$ can be explained by considering the polycrystalline nature of the sample. Grains with their easy axis parallel to the applied field are those that contribute to the spin flip. At $\mu_{0} H_{\mathrm{sf}}$, their spins are rapidly polarized along the field direction. In contrast, grains not orientated with their easy axis parallel to the field have their spins more slowly polarized along the field direction as the applied field increases. These spins contribute to the slow rise of $M(H)$ after $\mu_{0} H_{\text {sf }}$. By 7 T, $M(H)$ approaches $\approx 2 \mu_{\mathrm{B}}$ per $\mathrm{Ni}^{2+}$ ion. This is consistent with $M_{\text {sat }} \approx 2.29 \mu_{\mathrm{B}}$ per $\mathrm{Ni}^{2+}$ ion as expected from the $g$-factor extracted from the fit to $\chi^{-1}(T)$. The inset of Fig. 9(c) shows hysteretic behavior in $M(H)$ for $T<4 \mathrm{~K}$ similar to that observed in $\mathrm{Co}(\mathrm{NCS})_{2}$ (thiourea) $)_{2}$.
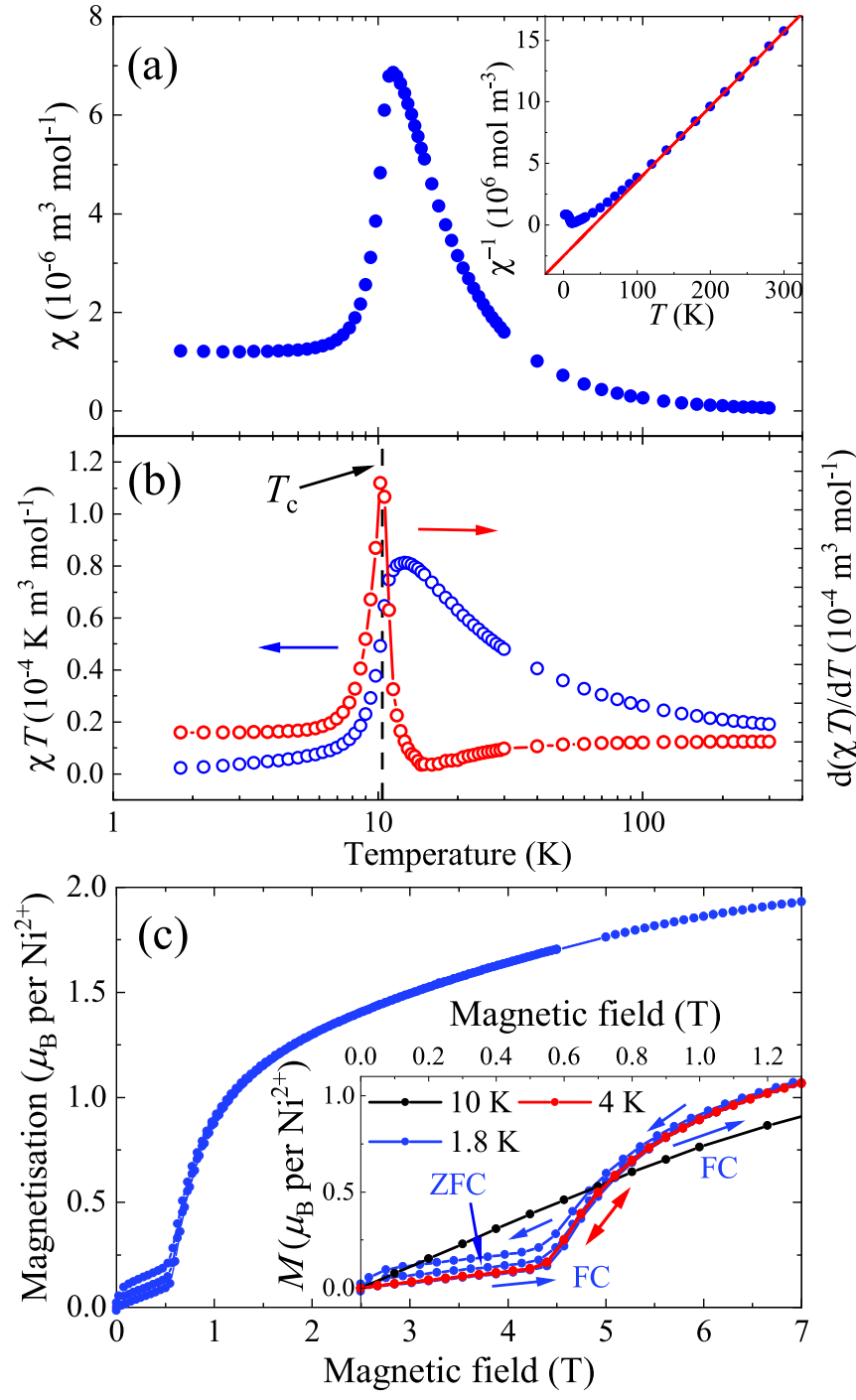

FIG. 9. (a) Powder magnetic susceptibility $\chi(T)$ data for $\mathrm{Ni}(\mathrm{NCS})_{2}$ (thiourea $)_{2}$ measured at $\mu_{0} H=0.1 \mathrm{~T}$. The inset shows $\chi^{-1}(T)$ fit to the Curie-Weiss model over the temperature range $100 \leqslant T \leqslant 300 \mathrm{~K}$. (b) $\chi(T)$ multiplied by temperature $[\chi T(T)]$ (blue, left-axis) and its derivative (red, right-axis) with critical temperature $T_{\mathrm{c}}$ marked with a dashed line. (c) Powder dc-field magnetization $M(H)$ for $\mathrm{Ni}(\mathrm{NCS})_{2}$ (thiourea $)_{2}$. The inset shows hysteretic behavior $T<4 \mathrm{~K}$ with $\mathrm{ZFC}$ and FC sweeps marked.

\section{E. Calculated exchange coupling constants}

To help validate the sign and strength of $J$ and $J^{\prime}$ determined from the magnetometry, the geometries optimized by DFT were used to calculate the magnetic superexchange coupling constants. Along each axis, the energy difference $(\Delta E)$ between ferromagnetic (FM) and antiferromagnetic (AFM) coupling was calculated for adjacent $M$ ions and used to obtain the sign and magnitude of the magnetic exchange interaction. As $\Delta E=E_{\mathrm{FM}}-E_{\mathrm{AFM}}, \Delta E<0$ is representative of AFM exchange. Values of $\Delta E$ were converted to exchange coupling by considering a single $J$ convention in the Hamiltonian (sum over unique exchange pathways); the results of these calculations are shown in Table II and Table SII [29]. We find that the primary exchange is FM in both materials 

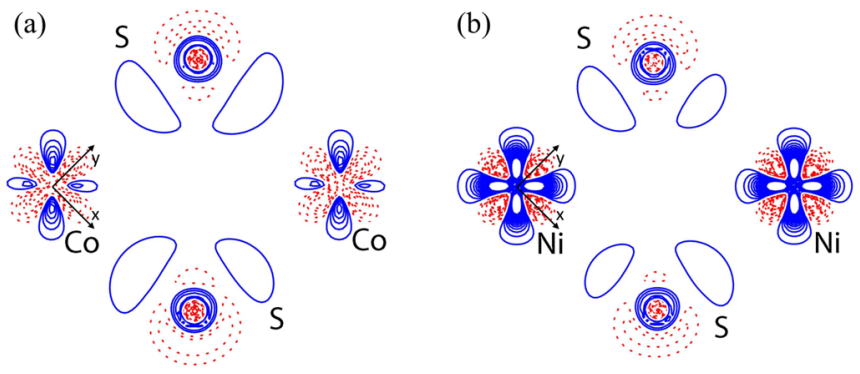

FIG. 10. Deformation electron-density maps for (a) $\mathrm{Co}(\mathrm{NCS})_{2}(\text { thiourea })_{2}$ and (b) $\mathrm{Ni}(\mathrm{NCS})_{2}$ (thiourea) $)_{2}$. Blue and red contours $\left(0.2 e \AA^{-3}\right)$ represent regions of electron charge-density excess and depletion, respectively.

(Table II), with $J=4.22 \mathrm{~K}$ for the Co species and $J=$ $78.13 \mathrm{~K}$ for the Ni compound (occurring along $a$; see Table SII [29]). Interchain exchange interactions in both compounds are on the order of $\left|J^{\prime}\right| \sim 0.1 \mathrm{~K}$ and shown in Table II. Weak AFM interchain exchange along $b$ is predicted in both compounds with FM exchange along $c$ predicted in the Ni species as outlined in Table SII [29]. Such a discrete change in sign of the exchange is difficult to verify experimentally. Due to the small magnitude of $J^{\prime}$, and the convergence criterion used for the energy calculations, we note that the calculated $J^{\prime}$ parameters are less reliable than those calculated for $J$. In addition, small changes in the lattice geometry can have statistically significant effects on such small energy differences. The calculated $J$ is roughly two and four orders of magnitude greater than $J^{\prime}$ for $\mathrm{Co}(\mathrm{NCS})_{2}$ (thiourea $)_{2}$ and $\mathrm{Ni}(\mathrm{NCS})_{2}$ (thiourea $)_{2}$, respectively (see Table II), supporting our argument of Q1D behavior.

\section{F. Charge and spin density}

DFT calculations and magnetometry data both suggest that the intrachain exchange in the Co species is significantly weaker than in the $\mathrm{Ni}$ species. To investigate the underlying mechanism responsible for the large difference in the values of $J$, electronic configurations of the $M$ sites, chargedensity maps, and calculated spin-density distributions were estimated; see [29] and [51-54] for details.

High-resolution x-ray diffraction data refined using the Hansen-Coppens multipolar model (MM) [55,56] were adopted to retrieve the electronic configuration and experimental charge-density distribution. The population of the fitted multipolar functions is effectively used to estimate the occupancy of $d$-orbitals' functions. Results for the Co material (see Table I) indicate that the ion resides in the high-spin $\left[t_{2 g}^{5}, e_{g}^{2}\right]$ electronic configuration. The experimental electronic configuration for $\mathrm{Co}^{2+}$ in $\mathrm{Co}(\mathrm{NCS})_{2}$ (thiourea) $)_{2}$ was validated by comparison with the MM refinement of the structure factor calculated from DFT [57,58]. In addition, DFT simulations allowed estimation of the electronic configuration of $\mathrm{Ni}^{2+}$ in $\mathrm{Ni}(\mathrm{NCS})_{2}$ (thiourea $)_{2}$ for which high-resolution x-ray data were not available (Table I). The occupancy of $d$-orbitals' functions suggests that the $\mathrm{Ni}$ ion is in the $\left[t_{2 g}^{6}, e_{g}^{2}\right]$ electronic configuration. In this case, the abundant occupancy of the $d_{x^{2}-y^{2}}$ and $d_{z^{2}}$ orbital functions can be an effect of electronspin density being partially delocalized onto the ligands.
TABLE I. Experimental (Exp. MM) and calculated (Calc. MM) $d$-orbitals' occupancy for $M(\mathrm{NCS})_{2}$ (thiourea $)_{2}$ as estimated from the multipolar model (MM). The $z$-axis was set along the unique $M-\mathrm{N}$ bond, while the $x$ - and $y$-axes were set along $M-\mathrm{S}$ bonds in the equatorial plane.

\begin{tabular}{llcc}
\hline \hline & \multicolumn{2}{c}{$M=\mathrm{Co}$} & $\begin{array}{c}M=\mathrm{Ni} \\
d \text {-orbital }\end{array}$ \\
\cline { 2 - 3 } & Exp. MM & Calc. MM & Calc. MM \\
\hline$z^{2}$ & $1.06(2)$ & 1.29 & 1.46 \\
$x z$ & $1.34(2)$ & 1.59 & 2.04 \\
$y z$ & $1.87(2)$ & 1.64 & 2.06 \\
$x^{2}-y^{2}$ & $1.42(2)$ & 1.30 & 1.44 \\
$x y$ & $1.87(2)$ & 1.63 & 2.04 \\
tot & $7.45(10)$ & 7.47 & 9.05 \\
\hline \hline
\end{tabular}

However, an occupancy slightly exceeding the formal one or two electrons is not unusual in MM refinement, since the multipoles are $d$-orbital shape functions freely refined against the structure factor, where occupancy values tending to two electrons represent fully occupied orbitals. The same issue concerns theoretical calculations that use orbital functions to compute a wave function from which individual orbital populations are extracted by projecting the crystal orbitals onto an atomic basis, and therefore they do not guarantee integer occupancy. Here, for the sake of a fair comparison, we adopted the very same kind of projection for experiment and calculations, namely multipolar expansion refined against measured or computed structure factors.

The valence electron-charge density, responsible for chemical bonding, is shown by deformation density maps in terms of regions of charge-density excess and depletion obtained from the difference between multipolar and spherical charge density. The different electronic configurations retrieved for $\mathrm{Co}(\mathrm{NCS})_{2}$ (thiourea $)_{2}$ and $\mathrm{Ni}(\mathrm{NCS})_{2}$ (thiourea $)_{2}$ are reflected in their deformation density maps. Figure 10 shows the deformation density in the equatorial plane (relevant for the intrachain magnetic exchange coupling). In both coordination polymers, the electron density is clearly depleted (dotted red contours) toward the S-ligands, the $d_{x^{2}-y^{2}}$ being singularly occupied in both materials. Conversely, there is a clear excess of electron-charge density located around the $\mathrm{Ni}$ ion in regions between S-ligands (blue contours) depicting the fully occupied $d_{x y}$ orbital. The Co ion has a third semioccupied orbital pointing between the ligands that leads to a reduction in the electron density along this direction, shown in Fig. 10(a) as a diminished blue contoured region along the $\mathrm{Co} \cdots \mathrm{Co}$ (through-space) direction.

Comparing bond lengths alone is not sufficient to establish the equivalence of interactions in isostructural compounds, as different ions have, for instance, different ionic radii, which play an important role in defining the strength and nature of the chemical bonds. Therefore, quantum theory of atoms in molecules (QTAIM) [59] was applied to find bond trajectories and analyze the properties of the electron-charge density at the bond critical points (bcp). Thus, electron-density-based bond properties allow us to quantitatively compare chemical bonds. The electron density and its Laplacian at the bcp have equivalent values in corresponding $M$-ligand interactions 
TABLE II. Comparison of the intrachain $J$, interchain $J^{\prime}$, and SIA $D$ determined experimentally $\left[a=\chi(T), b=M(H), c=C_{\text {mag }}(T)\right]$ and via DFT calculations. Negative exchange values indicate AFM interactions, and negative $D$ values indicate easy-axis behavior. DFT values were calculated by considering a single $J$ convention in the Hamiltonian where summations are made over unique exchange pathways.

\begin{tabular}{|c|c|c|c|c|c|c|}
\hline & \multicolumn{3}{|c|}{$\mathrm{Co}(\mathrm{NCS})_{2}(\text { thiourea })_{2}$} & \multicolumn{3}{|c|}{$\mathrm{Ni}(\mathrm{NCS})_{2}(\text { thiourea })_{2}$} \\
\hline & $J(\mathrm{~K})$ & $J^{\prime}(\mathrm{K})$ & $D(\mathrm{~K})$ & $J(\mathrm{~K})$ & $J^{\prime}(\mathrm{K})$ & $D(\mathrm{~K})$ \\
\hline Expt. & $3.62(7)^{\mathrm{a}}$ & $-0.3(1)^{b}$ & $\sim-100^{\mathrm{a}}$ & $\sim 100^{\mathrm{a}}$ & $-0.13(1)^{\mathrm{b}}$ & $\sim-10^{\mathrm{c}}$ \\
\hline DFT & 4.22 & -0.1 & & 78.13 & -0.1 & \\
\hline
\end{tabular}

(Table SV [29]), establishing that these interactions are indeed analogous in the two materials. Moreover, these quantities emphasize differences between bonds, e.g., the electron density at the $M-\mathrm{N}$ bond critical point is twice that of the $M-\mathrm{S}$ bonds, confirming strongly anisotropic octahedral environments in both materials. Properties at the $M \cdots M$ bcp are appreciably different, and no bond trajectory and corresponding bcp are found in $\mathrm{Ni}(\mathrm{NCS})_{2}$ (thiourea $)_{2}$. Some covalent character of the $M$-ligand interactions is shown by the QTAIM integrated charges in Table SIII [29], where the formal oxidation states of the $\mathrm{M}^{2+}$ and $\mathrm{NCS}^{-}$ions are reduced due to charge sharing in the bonds, a clear sign of deviation from purely ionic interactions. Likewise, a slightly positive charge on the thiourea ligand (formally neutral) is indicative of a ligand to metal $\sigma$-donation mechanism.

Electronic configurations and deformation density maps inferred the possible magnetic superexchange pathways in the materials, establishing that in $\mathrm{Co}(\mathrm{NCS})_{2}$ (thiourea $)_{2}$, a through-space magnetic interaction is available, whereas in $\mathrm{Ni}(\mathrm{NCS})_{2}$ (thiourea $)_{2}$ it is absent. The calculated electron spin-density distributions for the two compounds follow explicitly the differences in their electronic configurations. In $\mathrm{Co}(\mathrm{NCS})_{2}$ (thiourea $)_{2}$, the calculated spin-density distribution, centered around the Co ion, is quite spherical, as shown in Fig. 11(a). Here, the spin-density is concentrated not only toward the ligands but also directly along the $a$-axis, permitting two possible exchange pathways between adjacent $\mathrm{Co}$ ions: a through-bond interaction along the two $\mathrm{Co}-\mathrm{S}-\mathrm{Co}$ bridges, and a through-space Co... Co exchange interaction. The latter must be AFM as it is a result of bond-formation, which is subject to the Pauli exclusion principle. In contrast, the spin-density at the $\mathrm{Ni}$ sites is polarized only along the ligands; see Fig. 11(b). In this case, the spin-density is concentrated exclusively along the $\mathrm{Ni}-\mathrm{S}-\mathrm{Ni}$ pathways and toward the $\mathrm{Ni}-\mathrm{N}$ bond. Therefore, $\mathrm{Ni}(\mathrm{NCS})_{2}$ (thiourea $)_{2}$ can
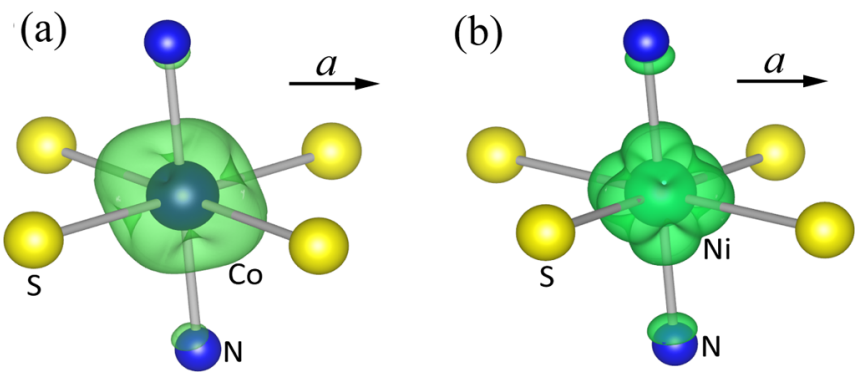

FIG. 11. Calculated spin-density maps for (a) $\mathrm{Co}(\mathrm{NCS})_{2}$ (thiourea $)_{2}$ and (b) $\mathrm{Ni}(\mathrm{NCS})_{2}$ (thiourea) $)_{2}$ plotted at the isovalue of $0.005 e$ per $\AA^{3}$. Spin-density regions are outlined as $3 \mathrm{D}$ green contours. only exploit the $\mathrm{Ni}-\mathrm{S}-\mathrm{Ni}$ through-bond interactions and, unlike $\mathrm{Co}(\mathrm{NCS})_{2}$ (thiourea $)_{2}$, has no through-space magnetic exchange.

\section{DISCUSSION}

Table II shows the values of $J, J^{\prime}$, and $D$ determined experimentally for both the $\mathrm{Ni}$ and Co compounds. An axial compression of the $M \mathrm{~S}_{4} \mathrm{~N}_{2}$ octahedra results in Ising-like anisotropy in both materials. As is typical (see [60]), the anisotropy energy in the Co material is found to be considerably larger than that in the Ni system.

Both compounds exhibit FM intrachain exchange, which is an order of magnitude greater in the $\mathrm{Ni}$ material than in the Co system. The electronic configurations, by identifying the semioccupied (magnetic) orbitals shown in Table I, determine that in $\mathrm{Ni}(\mathrm{NCS})_{2}$ (thiourea $)_{2}$ only a through-bond $M-\mathrm{S}-M$ exchange is possible, whereas in $\mathrm{Co}(\mathrm{NCS})_{2}$ (thiourea $)_{2}$ both through-bond $M-\mathrm{S}-M$ and through-space $M \cdots M$ magnetic interactions are available. As a result, in both materials, spin-density extends along the thiourea bibridges (Fig. 11), giving rise to strong FM exchange along the $M-\mathrm{S}-M$ pathways. However, the spin-density in the Co compound also extends directly along the $a$-axis, which leads to an additional AFM through-space interaction between Co ions. Competition between the two exchange pathways reduces the resultant intrachain exchange and explains the lower FM $J$ in the Co system. This highlights the sometimes subtle ways in which the choice of transition-metal ion affects the effective exchange strength. DFT calculations estimate the average interchain exchange to be around $-0.1 \mathrm{~K}$ in both compounds, in good agreement with magnetometry measurements.

$\mathrm{Ni}(\mathrm{NCS})_{2}$ (thiourea $)_{2}$ can be compared to the related material $\mathrm{NiCl}_{2}$ (thiourea) $)_{4}$ (DTN), an AFM-coupled spin chain that contains two field-induced phase transitions that may belong to the universality class of BEC [10,23]. Exchanging the $\mathrm{Cl}^{-}$ ion for the $\mathrm{NCS}^{-}$ion causes a structural change from I4 for DTN [23] to $P \overline{1}$ in the present case, and the change in the local $\mathrm{Ni}$ (II) environment, from $\mathrm{NiS}_{4} \mathrm{Cl}_{2}$ to $\mathrm{NiS}_{4} \mathrm{~N}_{2}$, has a strong effect on the SIA. The equatorial $\mathrm{Ni}-\mathrm{S}$ bond lengths are similar in both systems: $2.46 \AA$ for DTN and an average value of 2.57(3) $\AA$ for $\mathrm{Ni}(\mathrm{NCS})_{2}$ (thiourea $)_{2}$. However, the octahedra in DTN have a slight axial elongation with an equatorial to axial bond-length ratio of 0.98 , while in $\mathrm{Ni}(\mathrm{NCS})_{2}$ (thiourea) $)_{2}$ the octahedra are axially compressed with a bond-length ratio of 1.26. This results in the Ising-like SIA of $D \sim-10 \mathrm{~K}$, compared to the easy-plane SIA of $+8.12(4) \mathrm{K}$ observed in DTN [10].

The lack of spatial-inversion-symmetry in DTN results in a net electric polarization along the $c$ direction [61]. In contrast, $\mathrm{Ni}(\mathrm{NCS})_{2}$ (thiourea $)_{2}$ does possess a center 
of inversion symmetry, so we expect no net electric polarization.

The structure and SIA of DTN is such that AFM exchange is mediated along linear $\mathrm{Ni}-\mathrm{Cl} \cdots \mathrm{Cl}-\mathrm{Ni}$ pathways $[J=-1.74(3) \mathrm{K}]$ propagating along the $c$-axis. Within the $a b$-plane, nonbridging thiourea ligands keep the magnetic ions well separated (adjacent $\mathrm{Ni}$ sites are $9.595 \AA$ apart), which results in weak AFM $J^{\prime}=-0.17(1) \mathrm{K}$, possibly taking advantage of $\mathrm{Ni}-\mathrm{Cl} \cdots\left(\mathrm{H}_{2} \mathrm{~N}\right)_{2}-\mathrm{C}-\mathrm{S}-\mathrm{N}$ superexchange pathways.

For $\mathrm{Ni}(\mathrm{NCS})_{2}$ (thiourea $)_{2}$, the thiourea ligands are no longer terminal but now connect adjacent Ni sites along $a$ via $\mathrm{Ni}-\mathrm{S}-\mathrm{Ni}$ bibridges, which mediate the large FM exchange along $a$. The thiourea ligands still keep adjacent $\mathrm{Ni}$ sites well separated along $b$ at $7.527 \AA$, and H-bonding between the ligands mediates weak AFM $J^{\prime}$. Although Ni-NCS$\mathrm{Ni}$ bond pathways have been shown to effectively mediate magnetic exchange interactions in similar compounds [62], we find here that the NCS ligands are terminal and support only weak AFM $J^{\prime}$ via H-bonds along $c$. Thus, while DTN and $\mathrm{Ni}(\mathrm{NCS})_{2}$ (thiourea $)_{2}$ have highly similar equatorial environments, the dramatic structural change, invoked by substitution of the axial $\mathrm{Cl}^{-}$for $\mathrm{NCS}^{-}$, leads to drastically different magnetic properties, from a Q1D $X Y$-like AFM ground state exhibiting QCP behavior (DTN), to that of an FM coupled chain of Ising spins in $\mathrm{Ni}(\mathrm{NCS})_{2}$ (thiourea $)_{2}$.

Similarly, $\mathrm{Co}(\mathrm{NCS})_{2}$ (thiourea $)_{2}$ can be compared to the $S=3 / 2$ analog of DTN, $\mathrm{CoCl}_{2}$ (thiourea) 4 (DTC) [63]. DTC displays antiferromagnetic order below approximately $1 \mathrm{~K}$ that can be suppressed by magnetic fields of around $2 \mathrm{~T}$, and somewhat surprisingly, the observed susceptibility and magnetization are largely isotropic [61,64]. Swapping the $\mathrm{Cl}^{-}$for $\mathrm{NCS}^{-}$ion results in a structural change, now from $P 4_{2} / n$ to $P \overline{1}$. Average $\mathrm{Co}-\mathrm{S}$ bond lengths in DTC and $\mathrm{Co}(\mathrm{NCS})_{2}$ (thiourea $)_{2}$ are equal to within errors at 2.53(3) and 2.57(3) $\AA$, respectively. The local $\mathrm{CoS}_{4} \mathrm{~N}_{2}$ environment is compressed along the axial $\mathrm{N}-\mathrm{Co}-\mathrm{N}$ bond such that the ratio of the equatorial $\mathrm{Co}-\mathrm{S}$ to axial $\mathrm{Co}-\mathrm{N}$ bond is 1.28 , leading to a large Ising-like SIA $D \sim-100 \mathrm{~K}$. This contrasts with the isotropic behavior seen in DTC, which also possesses a slight axial compression, with a bond-length bond ratio of 1.02. Analogous to the comparison of the $\mathrm{Ni}$ species and DTN above, thiourea ligands form $\mathrm{Co}-\mathrm{S}-\mathrm{Co}$ bibridges in $\mathrm{Co}(\mathrm{NCS})_{2}$ (thiourea $)_{2}$ that mediate strong FM exchange along the $a$-axis, while the chains are well separated along $b$. Again, NCS ligands are terminal and mediate weak AFM interactions via $\mathrm{H}$-bond networks along the $c$-axis.

$\mathrm{Co}(\mathrm{NCS})_{2}$ (thiourea $)_{2}$ can also be compared to the archetype transverse-field Ising chain material $\mathrm{CoNb}_{2} \mathrm{O}_{6}$, with both possessing strong Ising-like SIA with FM $J$ and weak AFM $J^{\prime}$ [8]. Interchain exchange interactions induce a transition to long-range AFM order in both, at $T_{\mathrm{c}}=2.9 \mathrm{~K}$ in $\mathrm{CoNb}_{2} \mathrm{O}_{6}$ [65] and $T_{\mathrm{c}}=6.82(5) \mathrm{K}$ in $\mathrm{Co}(\mathrm{NCS})_{2}$ (thiourea) $)_{2}$. The lower $T_{\mathrm{c}}$ for $\mathrm{CoNb}_{2} \mathrm{O}_{6}$ indicates a more ideal 1D system, with $J^{\prime} \sim 0.01 \mathrm{~K}$ [66] compared to $0.1 \mathrm{~K}$ for
$\mathrm{Co}(\mathrm{NCS})_{2}$ (thiourea $)_{2}$. In $\mathrm{CoNb}_{2} \mathrm{O}_{6}$, a critical field applied perpendicular to the Ising axis breaks the 3D-AFM order, pushing it through a QCP as the material enters a quantum paramagnetic state $[8,9]$. Because application of a transverse field consistently shattered $\mathrm{Co}(\mathrm{NCS})_{2}$ (thiourea $)_{2}$ single crystals, we are as yet unable to provide evidence of similar quantum-critical behavior in our material.

\section{CONCLUSION}

In summary, we find that $M(\mathrm{NCS})_{2}$ (thiourea $)_{2}$, where $M=\mathrm{Ni}(\mathrm{II})$ or $\mathrm{Co}(\mathrm{II})$, both behave as Q1D chains with ferromagnetic intrachain exchange $J$, weak antiferromagnetic interchain interactions $J^{\prime}$, and Ising-like single-ion anisotropy $(D<0)$. At low temperature, long-range AFM ordering is observed in both materials as confirmed by heat capacity, magnetometry, and $\mu^{+} \mathrm{SR}$ measurements. The considerable difference in the magnitude of $J$ between the two compounds is due to their electronic configurations, where semioccupied orbitals are responsible for the different spin-density distributions, highlighting the prominent role of the transitionmetal-ion in promoting Q1D behavior. We find that the magnetic properties of the materials discussed here are very different from those observed in the chemically related quantum magnets DTN and DTC, due to significant structural changes induced by substitution of the axial $\mathrm{Cl}^{-}$halide ion for the $\mathrm{NCS}^{-}$ion.

Data presented in the paper resulting from the UK effort will be made available [67].

\section{ACKNOWLEDGMENTS}

This project has received funding from the European Research Council (ERC) under the European Union's Horizon 2020 research and innovation program (Grant Agreement No. 681260). Work at EWU was supported by NSF Grant No. DMR-1703003. NSF's ChemMatCARS Sector 15 is principally supported by the Divisions of Chemistry (CHE) and Materials Research (DMR), NSF, under Grant No. NSF/CHE-1834750. R.S. acknowledges support from the Swiss National Science Foundation (Project No. Nr.P2 BEP2_188253). This work is supported by the EPSRC (under Grants No. EP/N023803/1 and No. EP/N024028/1). The National High Magnetic Field Lab is funded by the U.S. National Science Foundation, U.S. Department of Energy and the State of Florida through Cooperative Grant No. DMR-1157490. Part of this work was carried out at the Swiss Muon Source, Paul Scherrer Institut, Switzerland, and we are grateful to Hubertus Luetkens for experimental assistance. F.X. would like to acknowledge the funding from the European Union's Horizon 2020 research and innovation program under the Marie Skłodowska-Curie Grant Agreement No. 701647. We thank Janice Musfeldt for useful discussions.
[1] S. Sachdev, Science 288, 475 (2000).

[2] A. Vasiliev, O. Volkova, E. Zvereva, and M. Markina, npj Quantum Mater. 3, 18 (2018).
[3] T. Giamarchi, C. Rüegg, and O. Tchernyshyov, Nat. Phys. 4, 198 (2008).

[4] F. D. M. Haldane, Phys. Rev. Lett. 50, 1153 (1983). 
[5] L. Savary and L. Balents, Rep. Prog. Phys. 80, 016502 (2016). [6] J. M. Kosterlitz and D. J. Thouless, J. Phys. C 6, 1181 (1973).

[7] V. Zapf, M. Jaime, and C. D. Batista, Rev. Mod. Phys. 86, 563 (2014).

[8] A. W. Kinross, M. Fu, T. J. Munsie, H. A. Dabkowska, G. M. Luke, S. Sachdev, and T. Imai, Phys. Rev. X 4, 031008 (2014).

[9] R. Coldea, D. A. Tennant, E. M. Wheeler, E. Wawrzynska, D. Prabhakaran, M. Telling, K. Habicht, P. Smeibidl, and K. Kiefer, Science 327, 177 (2010).

[10] V. S. Zapf, D. Zocco, B. R. Hansen, M. Jaime, N. Harrison, C. D. Batista, M. Kenzelmann, C. Niedermayer, A. Lacerda, and A. Paduan-Filho, Phys. Rev. Lett. 96, 077204 (2006).

[11] S. A. Zvyagin, J. Wosnitza, C. D. Batista, M. Tsukamoto, N. Kawashima, J. Krzystek, V. S. Zapf, M. Jaime, N. F. Oliveira, and A. Paduan-Filho, Phys. Rev. Lett. 98, 047205 (2007).

[12] J. Brambleby, P. A. Goddard, R. D. Johnson, J. Liu, D. Kaminski, A. Ardavan, A. J. Steele, S. J. Blundell, T. Lancaster, P. Manuel, P. J. Baker, J. Singleton, S. G. Schwalbe, P. M. Spurgeon, H. E. Tran, P. K. Peterson, J. F. Corbey, and J. L. Manson, Phys. Rev. B 92, 134406 (2015).

[13] P. R. Hammar, M. B. Stone, D. H. Reich, C. Broholm, P. J. Gibson, M. M. Turnbull, C. P. Landee, and M. Oshikawa, Phys. Rev. B 59, 1008 (1999).

[14] F. M. Woodward, A. S. Albrecht, C. M. Wynn, C. P. Landee, and M. M. Turnbull, Phys. Rev. B 65, 144412 (2002).

[15] P. A. Goddard, J. Singleton, P. Sengupta, R. D. McDonald, T. Lancaster, S. J. Blundell, F. L. Pratt, S. Cox, N. Harrison, J. L. Manson, H. I. Southerland, and J. A. Schlueter, New J. Phys. 10, 83025 (2008).

[16] P. A. Goddard, J. L. Manson, J. Singleton, I. Franke, T. Lancaster, A. J. Steele, S. J. Blundell, C. Baines, F. L. Pratt, R. D. McDonald, O. E. Ayala-Valenzuela, J. F. Corbey, H. I. Southerland, P. Sengupta, and J. A. Schlueter, Phys. Rev. Lett. 108, 077208 (2012).

[17] J. Liu, S. Kittaka, R. D. Johnson, T. Lancaster, J. Singleton, T. Sakakibara, Y. Kohama, J. van Tol, A. Ardavan, B. H. Williams, S. J. Blundell, Z. E. Manson, J. L. Manson, and P. A. Goddard, Phys. Rev. Lett. 122, 057207 (2019).

[18] B. M. Huddart, J. Brambleby, T. Lancaster, P. A. Goddard, F. Xiao, S. J. Blundell, F. L. Pratt, J. Singleton, P. MacChi, R. Scatena, A. M. Barton, and J. L. Manson, Phys. Chem. Chem. Phys. 21, 1014 (2019).

[19] R. C. Williams, W. J. A. Blackmore, S. P. M. Curley, M. R. Lees, S. M. Birnbaum, J. Singleton, B. M. Huddart, T. J. Hicken, T. Lancaster, S. J. Blundell, F. Xiao, A. Ozarowski, F. L. Pratt, D. J. Voneshen, Z. Guguchia, C. Baines, J. A. Schlueter, D. Y. Villa, J. L. Manson, and P. A. Goddard, Phys. Rev. Research 2, 013082 (2020).

[20] G. Yagupsky, R. H. Negrotti, and R. Levitus, J. Inorg. Nucl. Chem. 27, 2603 (1965).

[21] C. Puglisi and R. Levitus, J. Inorg. Nucl. Chem. 29, 1069 (1967).

[22] M. Nardelli, G. F. Gasparri, G. G. Battistini, and P. Domiano, Acta Crystallogr. 20, 349 (1966).

[23] B. N. Figgis and P. A. Reynolds, J. Chem. Soc., Dalton Trans., 125 (1986).

[24] J. B. Goodenough, Phys. Rev. 100, 564 (1955).

[25] J. Kanamori, J. Phys. Chem. Solids 10, 87 (1959).
[26] R. Ivaniková, R. Boča, L. Dlháň, H. Fuess, A. Mašlejová, V. Mrázová, I. Svoboda, and J. Titiš, Polyhedron 25, 3261 (2006).

[27] J. Titiš, R. Boča, L. Dlháň, T. Durčeková, H. Fuess, R. Ivaniková, V. Mrázová, B. Papánková, and I. Svoboda, Polyhedron 26, 1523 (2007).

[28] J. Titiš and R. Boča, Inorg. Chem. 50, 11838 (2011).

[29] See Supplemental Material at http://link.aps.org/supplemental/ 10.1103/PhysRevMaterials.5.034401 for experimental details and further analysis of $\mu^{+} \mathrm{SR}$, heat capacity, and SCXRD.

[30] F. L. Pratt, Physica B 289-290, 710 (2000).

[31] A. J. Steele, T. Lancaster, S. J. Blundell, P. J. Baker, F. L. Pratt, C. Baines, M. M. Conner, H. I. Southerland, J. L. Manson, and J. A. Schlueter, Phys. Rev. B 84, 064412 (2011).

[32] F. Xiao, J. S. Möller, T. Lancaster, R. C. Williams, F. L. Pratt, S. J. Blundell, D. Ceresoli, A. M. Barton, and J. L. Manson, Phys. Rev. B 91, 144417 (2015).

[33] T. Lancaster, S. Blundell, M. Brooks, F. Pratt, and J. Manson, Physica B 374, 118 (2006).

[34] J. L. Manson, C. M. Brown, Q. Huang, J. A. Schlueter, T. Lancaster, S. J. Blundell, J. Singleton, J. W. Lynn, and F. L. Pratt, Polyhedron 52, 679 (2013).

[35] J. Liu, P. A. Goddard, J. Singleton, J. Brambleby, F. Foronda, J. S. Möller, Y. Kohama, S. Ghannadzadeh, A. Ardavan, S. J. Blundell, T. Lancaster, F. Xiao, R. C. Williams, F. L. Pratt, P. J. Baker, K. Wierschem, S. H. Lapidus, K. H. Stone, P. W. Stephens, J. Bendix, T. J. Woods, K. E. Carreiro, H. E. Tran, C. J. Villa, and J. L. Manson, Inorg. Chem. 55, 3515 (2016).

[36] J. J. Binney, N. J. Dowrick, A. J. Fisher, and M. E. Newman, The Theory of Critical Phenomena: An Introduction to the Renormalization Group (Oxford University Press, Oxford, 1992).

[37] J. C. Lashley, M. F. Hundley, A. Migliori, J. L. Sarrao, P. G. Pagliuso, T. W. Darling, M. Jaime, J. C. Cooley, W. L. Hults, L. Morales, D. J. Thoma, J. L. Smith, J. Boerio-Goates, B. F. Woodfield, G. R. Stewart, R. A. Fisher, and N. E. Phillips, Cryogenics (Guildf) 43, 369 (2003).

[38] R. Honmura, Phys. Rev. B 30, 348 (1984).

[39] X. Feng and H. W. J. Blöte, Phys. Rev. E 81, 031103 (2010).

[40] L. Onsager, Phys. Rev. 65, 117 (1944).

[41] B. M. McCoy and T. T. Wu, The Two-Dimensional Ising Model (Harvard University Press, Cambridge, MA, 1973).

[42] P. Baran, M. Boča, R. Boča, A. Krutošíková, J. Miklovič, J. Pelikán, and J. Titiš, Polyhedron 24, 1510 (2005).

[43] J. Titiş and R. Boça, Inorg. Chem. 49, 3971 (2010).

[44] M. E. Fisher, Philos. Mag. 7, 1731 (1962).

[45] M. E. Fisher, J. Math. Phys. 4, 124 (1963).

[46] R. E. Greeney, C. P. Landee, J. H. Zhang, and W. M. Reiff, Phys. Rev. B 39, 12200 (1989).

[47] M. E. Lines, J. Chem. Phys. 55, 2977 (1971).

[48] F. Lloret, M. Julve, J. Cano, R. Ruiz-García, and E. Pardo, Inorg. Chim. Acta 361, 3432 (2008).

[49] M. Rams, Z. Tomkowicz, M. Böhme, W. Plass, S. Suckert, J. Werner, I. Jess, and C. Näther, Phys. Chem. Chem. Phys. 19, 3232 (2017).

[50] W. J. A. Blackmore, J. Brambleby, T. Lancaster, S. J. Clark, R. D. Johnson, J. Singleton, A. Ozarowski, J. A. Schlueter, Y.-S. Cheng, A. M. Arif, S. Lapidus, F. Xiao, R. C. Williams, S. J. Blundell, M. J. Pearce, M. R. Lees, P. Manuel, D. Y. Villa, J. A. Villa, J. L. Manson, and P. A. Goddard, New J. Phys. 21 093025 (2019) 
[51] P. Macchi, H.-B. Bürgi, A. S. Chimpri, J. Hauser, and Z. Gál, J. Appl. Crystallogr. 44, 763 (2011).

[52] Crysalispro, CrysAlisPRO, Oxford Diffraction /Agilent Technologies UK Ltd., Yarnton, England.

[53] G. M. Sheldrick, Acta Crystallogr., Sect. C 71, 3 (2015).

[54] A. Valkov, P. Macchi, L. J. Farrugia, C. Gatti, P. Mallinson, T. Richter, and T. Koritsanszky, XD2016 (2016), available at https://www.chem.gla.ac.uk/ louis/xd-home/.

[55] N. K. Hansen and P. Coppens, Acta Crystallogr., Sect. A 34, 909 (1978).

[56] A. Holladay, P. Leung, and P. Coppens, Acta Crystallogr., Sect. A 39, 377 (1983).

[57] R. Dovesi, R. Orlando, A. Erba, C. M. Zicovich-Wilson, B. Civalleri, S. Casassa, L. Maschio, M. Ferrabone, M. De La Pierre, P. D'Arco, Y. Noël, M. Causà, M. Rérat, and B. Kirtman, Int. J. Quantum Chem. 114, 1287 (2014).
[58] M. F. Peintinger, D. V. Oliveira, and T. Bredow, J. Comput. Chem. 34, 451 (2013).

[59] R. F. W. Bader, Chem. Rev. 91, 893 (1991).

[60] R. Boča, Coord. Chem. Rev. 248, 757 (2004).

[61] E. Mun, J. Wilcox, J. L. Manson, B. Scott, P. Tobash, and V. S. Zapf, Adv. Condens. Matter Phys. 2014, 512621 (2014).

[62] J. Palion-Gazda, B. Machura, F. Lloret, and M. Julve, Cryst. Growth Des. 15, 2380 (2015).

[63] J. E. O’Connor and E. L. Amma, Inorg. Chem. 8, 2367 (1969).

[64] H. Forstat, N. D. Love, J. McElearney, and H. Weinstock, Phys. Rev. 145, 406 (1966).

[65] W. Scharf, H. Weitzel, I. Yaeger, I. Maartense, and B. M. Wanklyn, J. Magn. Magn. Mater. 13, 121 (1979).

[66] T. Kunimoto, K. Nagasaka, H. Noriji, S. Luther, M. Motokawa, H. Ohta, T. Goto, S. M. Okubo, and K. Kohn, J. Phys. Soc. Jpn. 68, 1703 (1999).

[67] https://wrap.warwick.ac.uk/148301. 